Meta

Journal des traducteurs

Translators' Journal

\title{
Le doublage au Canada : politiques de la langue et langue des politiques
}

\section{Robert Paquin}

Volume 45, numéro 1, avril 2000

La traduction littéraire au Canada

Literary Translation in Canada

URI : https://id.erudit.org/iderudit/003779ar

DOI : https://doi.org/10.7202/003779ar

Aller au sommaire du numéro

Éditeur(s)

Les Presses de l'Université de Montréal

ISSN

0026-0452 (imprimé)

1492-1421 (numérique)

Découvrir la revue

Citer cet article

Paquin, R. (2000). Le doublage au Canada : politiques de la langue et langue des politiques. Meta, 45(1), 127-133. https://doi.org/10.7202/003779ar
Résumé de l'article

Quels sont les enjeux politiques, culturels et économiques auxquels fait face l'industrie du doublage au Canada? Peut-on parler d'acculturation du Canada par les États-Unis sans parler d'acculturation du Canada francophone par la France? Quel contrôle le Canada exerce-t-il? 


\title{
Le doublage au Canada: politiques de la langue et langue des politiques
}

\author{
ROBERT PAQUIN \\ Traducteur et poète
}

\begin{abstract}
RÉSUMÉ
Quels sont les enjeux politiques, culturels et économiques auxquels fait face l'industrie du doublage au Canada? Peut-on parler d'acculturation du Canada par les États-Unis sans parler d'acculturation du Canada francophone par la France? Quel contrôle le Canada exerce-t-il?
\end{abstract}

\begin{abstract}
What are the political, cultural and economic challenges facing the Canadian dubbing industry? Is it possible to discuss cultural assimilation of Canada by the USA without discussing that of French Canada by France? How much control does Canada have?

MOTS-CLÉS/KEYWORDS

doublage, cinéma, politiques linguistiques, Canada, traduction pour le cinéma
\end{abstract}

\section{Introduction}

Étant donné qu'il s'agit de cinéma et de télévision, on est tenté d'imaginer une confrontation dans la tradition du western. Il est midi, la rue est déserte, portes et fenêtres closes, le vent balaie des brindilles de paille, tandis que l'un des protagonistes apparaît sous un stetson poussiéreux à l'autre bout de la ville, la main planant audessus de son colt. L'autre cow-boy, lui aussi armé, est appuyé au chambranle de la porte du saloon et suit la progression de son adversaire d'un regard froid et sec. Qui est le bon, qui est le méchant? Qui sont ces frères ennemis?

Le Canada, ou plutôt le Québec, et la France, jusqu'ici alliés face au danger de l'acculturation par le géant américain, s'affrontent aujourd'hui, l'un armé d'un Décret et d'une Entente, et l'autre sans autre munition que sa volonté de survivre. Je crois cependant qu'il faut abandonner ce scénario mélodramatique qui ne donne pas une idée juste de la problématique. Essayons de brosser un tableau sommaire de la situation.

\section{Le Décret}

Le Décret nº 96-776 du 2 septembre 1996 stipule que:

L'exploitation d'une œuvre cinématographique doublée en langue française est subordonnée à l'obtention d'un visa distinct de celui délivré pour l'exploitation de l'œuvre dans la version originale.

Le visa d'exploitation en version doublée ne peut être accordé que si la version originale a obtenu le visa d'exploitation et si le doublage a été entièrement réalisé dans des studios situés sur le territoire français ou sur le territoire d'un autre État, membre de 
l'Union européenne ou Partie à l'accord sur l'Espace économique européen du 2 mai 1992. Toutefois, cette seconde condition n'est pas exigée pour les œuvres d'origine canadienne doublées au Canada ${ }^{1}$. (C’est moi qui souligne.)

Cette réglementation française, qui s'applique au doublage des films projetés dans des salles de cinéma, n'exclut à toutes fins utiles que les films doublés au Québec puisque, hormis la France, c'est le seul endroit où il y a doublage en français de films destinés aux salles. Voilà pour le cinéma.

\section{L'entente}

Quant à la télévision, les syndicats français des acteurs signaient une entente avec les principaux télédiffuseurs français, limitant la diffusion de productions doublées ailleurs qu'en France. Cette entente, qui a été reconduite durant plusieurs années, précisait que les trois sociétés nationales de télévision de l'époque s'engageaient «à n'acquérir les droits des téléfilms, séries et feuilletons télévisés ayant la nationalité d'un ou plusieurs pays extérieurs à la C.E.E. que si ces produits sont entièrement doublés sur le territoire français ${ }^{2} »$. (C’est moi qui souligne.)

Le doublage au Québec se voit donc limité... au Québec. Il s'agit d'un cas étrange de mesures protectionnistes adoptées par un pays dominant pour se protéger de la concurrence d'un plus petit. Selon une étude commandée par madame Louise Beaudoin, alors ministre de la Culture et des Communications du Québec, et effectuée par la Société de développement des entreprises culturelles (SODEC), «l'industrie du doublage au Québec représente un chiffre d'affaires annuel de 19 M\$, réparti entre une dizaine d'entreprises qui emploient près de 700 personnes, dont 500 comédiens. ${ }^{3}$ Selon l'évaluation de cette même étude qu'on appelle généralement le rapport Lampron, du nom du président de la SODEC, le chiffre d'affaires global annuel des entreprises de doublage en France serait de plus de 100 M\$. De plus, on établit à plus ou moins 2000 le nombre de comédiens doubleurs en France 4 . La France représente un marché de 60 millions de consommateurs francophones par rapport à 6 millions pour le Québec.

Au Canada, même si le doublage cinématographique et télévisuel ne se pratique qu'au Québec, les lois régissant la télévision relèvent du gouvernement fédéral, alors que le cinéma en salles est de juridiction provinciale. Quels sont les organismes concernés?

\section{Les institutions}

- Le Conseil de la radiodiffusion et des télécommunications canadiennes (CRTC $)^{5}$ a pour mission de «veiller à ce que le secteur des communications canadiennes contribue de façon juste et équitable à la prospérité économique, sociale et culturelle du pays, en ayant recours à la réglementation, à la supervision et à un dialogue ouvert avec le public ». Le CRTC a, entre autres, le pouvoir d'imposer aux diffuseurs des quotas de contenu canadien.

- Téléfilm Canada ${ }^{6}$ "est un organisme culturel fédéral voué au développement et à la promotion de l'industrie canadienne du film, de la télévision, de la vidéo et de produits multimédias ».

- $\quad$ Le Fonds canadien de télévision (FCT $)^{7}$ est « un programme de financement clé destiné à l'industrie canadienne de la télévision et du long métrage » et administré par Téléfilm Canada. 
- Le ministère de la Culture et des communications du Québec (MCC) ${ }^{8}$ compte les industries culturelles, donc l'industrie du doublage, parmi ses champs de compétence.

- La Société de développement des entreprises culturelles (SODEC) est une société d'État du gouvernement du Québec, relevant du ministère de la Culture et des Communications. La SODEC «a pour mandat de promouvoir et de soutenir, dans toutes les régions du Québec, l'implantation et le développement des entreprises culturelles, y compris les médias. Elle a aussi pour fonction de contribuer à accroître la qualité des produits et des services et la compétitivité de ceux-ci sur tous les marchés ${ }^{9} . »$

- L'Association québécoise des industries techniques du cinéma et de la télévision (AQITCT) regroupe les maisons de production et les studios qui font du doublage.

- L'Union des artistes (UDA) est le syndicat qui représente, entre autres, les comédiens qu'on trouve sur les plateaux de doublage au Québec. «Même si les activités de doublage constituent globalement une activité marginale pour les comédiens de l'UDA (2,5 M\$ sur des revenus globaux de $36 \mathrm{M} \$$ ), certains d'entre eux y puisent une part importante de leurs revenus ${ }^{10}$.»

- Au Canada, les traducteurs/adaptateurs (dialoguistes et sous-titreurs) responsables des textes qui servent au doublage et au sous-titrage ne sont véritablement regroupés dans aucune organisation spécifique. Sur une quinzaine de personnes qui se consacrent à ce travail, deux seulement font partie de l'Association des traducteurs et traductrices littéraires du Canada (ATTLC) ${ }^{11}$, dont le but est de promouvoir la traduction littéraire en général. En France, les dialoguistes sont représentés par le Syndicat National des Auteurs et Compositeurs. Soit dit en passant, la SOCAN (Société canadienne des auteurs, compositeurs et éditeurs de musique $)^{12}$ ne perçoit pas de droits d'auteurs au nom des traducteurs/adaptateurs, alors qu'en France, la SACEM (Société des auteurs, compositeurs et éditeurs de musique ${ }^{13}$ gère aussi les droits des dialoguistes.

\section{L'aide au doublage}

En 1989, à la demande de l'Union des artistes (UDA) d'établir des exigences minimales relativement aux émissions doublées au Canada et comparables à celles qu'il impose en matière de contenu canadien, le CRTC répliqua «qu'une telle pratique serait inefficace car elle pourrait entraîner un effet négatif sur la production d'émissions originales canadiennes, en ce que des fonds du budget de programmation canadienne pourraient être réaffectés aux dépenses en doublage. De plus, cette mesure constituerait un excès de réglementation et serait source d'inefficacité économique en incitant au doublage au Canada de nombreuses émissions déjà doublées en France. $\gg^{14}$ Le CRTC décida donc de ne pas imposer de quotas de doublage, en dépit du fait que les Français, eux, n'hésitent pas à doubler à nouveau les films déjà doublés en français au Québec. Ainsi, pour ne citer qu'un exemple, malgré l'excellente adaptation française du film La petite sirène de Walt Disney par Vincent Davy qui assurait également la direction du doublage, les Français ont refait leur propre version avec un autre texte et leurs propres comédiens. Peut-être n'est-ce donc pas une simple question d'économie? Quoi alors? La langue? Mais monsieur Davy est un Français qui a émigré au Canada à l'âge adulte.

\section{Soutien public à l'industrie du doublage}

Téléfilm Canada offre une aide financière pour le doublage et le sous-titrage de productions canadiennes, et exige que le doublage des productions qu'elle soutient soit effectué au Canada. En ce qui concerne la télévision, cette aide est administrée par le 
Fonds canadien de télévision (FCT). Cependant l'AQITCT et l'UDA regrettent que, depuis le début de l'année financière 1996-1997, ce soutien ne s'applique plus au doublage d'œuvres étrangères.

Selon M. Alain Ergas, directeur des acquisitions à la chaîne Télé-Québec, un documentaire d'une heure doublé au Québec, sans l'appui financier de Téléfilm, coûte deux fois plus cher qu'un documentaire déjà doublé en France. Le coût de revient des versions doublées au Québec est plus élevé parce qu'elles ne sont pratiquement jamais diffusées en dehors du Québec puisque le marché français nous est interdit. Le télédiffuseur québécois se voit donc dans l'obligation soit d'attendre qu'une chaîne française ait décidé de présenter une émission étrangère, britannique ou américaine par exemple, avant de l'acquérir et de la mettre à son programme, soit d'acquérir deux fois moins d'heures d'émissions et de les faire doubler pour son usage personnel, sans grand espoir de pouvoir les revendre ailleurs.

\section{Délais de doublage avantageux}

Pour Hollywood, le Canada fait partie du marché local, c'est-à-dire que les films américains sortent en même temps à Montréal ou ailleurs au Québec qu'à Los Angeles ou New York, alors que la projection du même film dans les salles européennes, même dans les grandes capitales, comme Paris, Bruxelles ou Berlin, peut être retardée de plusieurs mois. Le Québec ayant toujours le souci de protéger sa population d'une anglicisation dévorante et voulant éviter que les cinéphiles québécois ne soient obligés de voir les productions américaines en anglais, le ministère de la Culture et des Communications (MCC) a donc fait adopter un article de loi stipulant «que les films en langue autre que le français doivent être disponibles en français dans les 45 jours à compter de la date de la première présentation du film en salle ${ }^{15} »$. L'article 83 de la Loi sur le cinéma ne précise pas si cette version française doit être doublée ou sous-titrée, ni si elle doit être produite au Québec ou ailleurs, mais étant donné que les dates de sortie ne sont pas les mêmes en Europe et que le public québécois préfère en général le doublage au sous-titrage, ce facteur a contribué à l'essor de l'industrie du doublage au Québec. Selon M. Christian Verber de la SODEC, alors qu'en 1990 seulement $34 \%$ des films étrangers projetés dans les salles du Québec étaient doublés au Québec, en 1998, cette proportion atteignait $58 \%$. M. Verber attribue ce changement à l'application de la loi. Le rapport Lampron note cependant qu'il est possible que les majors (les grandes maisons de production de Hollywood) décident "d'aligner les sorties en Europe sur les sorties nord-américaines» et qu'alors elles «optent de plus en plus pour un doublage français susceptible de desservir l'ensemble des territoires francophones ${ }^{16} »$. Cette éventualité suscite certaines inquiétudes. "On n'a qu'à penser au marché de la télévision où l'industrie québécoise du doublage ne parvient plus à récupérer la place marquante qu'elle détenait jusqu'au début des années 1980 », peut-on lire dans le rapport Lampron ${ }^{17}$.

\section{Crédits d'impôt}

Une autre forme d'aide à l'industrie du doublage au Canada prend la forme de crédits d'impôt. En effet, «lorsque les coûts de doublage ont été acceptés au budget de production par les différents investisseurs, les producteurs peuvent avoir accès aux 
crédits d'impôt fédéral et québécois pour cette portion du budget ${ }^{18}{ }$. Jusqu'à récemment, cette aide indirecte ne s'appliquait qu'au doublage de productions canadiennes. Dans le but de favoriser le doublage au Québec et le développement de l'industrie cinématographique, le rapport Lampron propose cependant qu'un crédit d'impôt soit accordé aux entreprises de doublage québécoises tant par le gouvernement provincial que par le gouvernement canadien pour toutes leurs activités de doublage ${ }^{19}$. Il est encore trop tôt cependant pour évaluer les effets de ces mesures destinées à encourager et soutenir une industrie que plusieurs croient déjà moribonde.

\section{À qui la faute?}

Il est tentant d'accuser les «maudits Français» et plusieurs ne se gênent pas pour le faire: "Comment se fait-il que nous, on comprend leur dialecte, alors qu'eux, font semblant de ne pas nous comprendre?» ou «Pourquoi serions-nous obligés d'entendre des voyous du Bronx s'engueuler en argot parisien?» Le rapport Lampron mentionne cette préférence du public québécois pour des films doublés ici. Pourtant, la dernière des conclusions du rapport est d' " encourager les entreprises québécoises à partager les travaux de doublage avec les entreprises françaises ${ }^{20} »$. On peut se demander de quel genre de partage il peut s'agir. Quelle étape du processus de doublage confierait-on à la France? En simplifiant, on pourrait dire que le doublage comporte trois étapes: la détection, la traduction/adaptation et l'enregistrement des voix. Or il est bien évident que le rapport ne propose pas de remplacer les voix des comédiens québécois par des voix françaises. Le lobby de l'UDA est trop puissant pour que le gouvernement songe à le contourner. Il reste la détection et la traduction/adaptation. La détection est un travail technique qui pourrait effectivement être réalisé n'importe où, en France ou ailleurs. D'ailleurs, un bon détecteur peut reconnaître et transcrire des langues qu'il ne parle pas.

Quant à la traduction, on le sait, elle a toujours été la parente pauvre et sousestimée du processus de création, la dernière à être embauchée et la première à se faire «virer». Comme on l'a vu, contrairement aux comédiens, les traducteurs/adaptateurs/dialoguistes canadiens ne sont pas représentés par une organisation syndicale comme leurs collègues français. Pourtant, sans leur texte, les comédiens n'auraient rien à dire. Et si la collaboration avec les entreprises françaises envisagée par le rapport Lampron se fait au détriment des traducteurs canadiens, et qu'on fait traduire un certain nombre de films en France pour ensuite les faire interpréter par des comédiens canadiens, rien n'aura été changé du point de vue du public. On entendra des voix d'acteurs québécois parlant avec un accent français (qu'ils peuvent très bien imiter) et s'exprimant en argot parisien. C'est le texte qui fait la différence, pas la voix.

\section{Traduire pour qui ?}

Si je traduis une série télévisée pour exportation en Afrique francophone, comme je l'ai fait ces dernières années avec deux séries policières et deux feuilletons allemands, je choisis les termes et les expressions en fonction d'un public autre que si je traduis des dessins animés pour diffusion au Canada. J'évite d'employer des expressions locales qui n'ont pas de résonance dans un autre contexte. Par exemple, je parlerai d'une 
ambulance ou d'un service ambulancier, plutôt que d'Urgence Santé ou du SAMU (Service ambulancier médical d'urgence).

Le traducteur/adaptateur/dialoguiste français ne s'embête généralement pas de telles nuances. Il s'adresse à un public de 60 millions de téléspectateurs français habitués à la norme parisienne et ne se soucie nullement de ce qui se dit dans les «colonies» ou dans les pays francophones limitrophes, comme la Belgique ou la Suisse, d'où une forme d'impérialisme ou d'acculturation par la métropole française qui impose SA langue. Dans Parler croquant (1978), Claude Duneton a déjà dénoncé cet impérialisme qui s'exprime même à l'intérieur de l'Hexagone au détriment des dialectes et langues régionales.

Tout comme le cinéma hollywoodien omniprésent qui tend à niveler les différences culturelles et à les ramener à la vision américaine ${ }^{21}$, l'imposition sur les écrans canadiens d'un doublage réalisé en France nuit au développement de notre industrie nationale du doublage en plus d'être une source de frustration pour le public.

\section{Une solution peu économique}

Le président de l'Union des artistes, Pierre Curzi, exposait récemment le plan d'action de son organisme ${ }^{22}$. Pour lui, il s'agit de lancer une opération de séduction afin de convaincre les majors de l'avantage de faire doubler leurs films au Québec, tout en tâchant d'obtenir le support du public et du gouvernement. Il est bien évident que la sauvegarde de l'identité culturelle du Québec (et du Canada) va nécessiter des sacrifices, et il serait illusoire de croire qu'Hollywood consentira à ces sacrifices. Tant que les produits cinématographiques et télévisuels seront considérés uniquement comme des produits devant rapporter des profits, la culture sera perdante. Il faut donc que les gouvernements admettent la nécessité d'imposer des règles et des limites. Il faut également qu'ils soient prêts à assumer le coût collectif du maintien de ces limites. La solution n'est sûrement pas dans un affrontement qui ne servirait qu'à briser des alliances déjà fragilisées par la distance.

Nos deux protagonistes du début auraient donc avantage à ranger leurs colts et à se retrouver au saloon pour boire une bière ensemble et chercher à s'entendre sur la façon la plus efficace de se protéger de la tornade qui menace à l'horizon. Ils peuvent apprendre l'un de l'autre et s'appuyer dans leurs efforts d'obtention de soutien de leurs gouvernements respectifs pour construire des clôtures et des abris qui assureront le maintien de leur culture propre.

\section{NOTES}

1. Décret $\mathrm{n}^{\circ}$ 96-776 du 2 septembre 1996 pris pour l'application du Code de l'industrie cinématographique et relatif à la classification des œuvres cinématographiques.

2. L'industrie du doublage: Consolidation et nouveaux marchés, août 1998, p. 20, <http://www.mcc. gouv.qc.ca/pubprog/rapports/tablemd.htm>

3. Ibid., p. 3.

4. Ibid., p. 18.

5. <http://www.crtc.gc.ca/>

6. <http://www.telefilm.gc.ca/fr/intro.htm>

7. <http://www.fondscanadiendetele.ca/frn/index.htm>

8. <http://www.mcc.gouv.qc.ca/>

9. <http://www.mcc.gouv.qc.ca/pubprog/pasodec/intsodec.htm>

10. L'industrie du doublage, op. cit., p. 3. 
11. <http://www.geocities.com/Athens/Oracle/9070/>

12. <http://www.socan.ca/fr/home.html>

13. <http ://www.sacem.fr/index1.html >

14. CRTC-Radiodiffusion, Ottawa, le 13 août 1992, Avis public CRTC 1992-53.

15. L'industrie du doublage, op. cit., p. 9.

16. Ibid., p. 13.

17. Ibid.

18. Ibid., p. 15.

19. Ibid., p. 36

20. Ibid., p. 38.

21. Voir Le rêve américain, documentaire de l'Office national du film en cours de réalisation.

22. Le Devoir, 19 mai 1999, p. B 10.

\section{RÉFÉRENCE}

Duneton, Claude (1978): Parler croquant, Paris, Stock. 